Notfall Rettungsmed 2023 $\cdot 26: 93-100$ https://doi.org/10.1007/s10049-022-00985-2 Angenommen: 17. Januar 2022 Online publiziert: 18. Februar 2022

(c) The Author(s), under exclusive licence to Springer Medizin Verlag $\mathrm{GmbH}$, ein Teil von Springer Nature 2022

\section{Pandemienotfallhelfer - gestärkte Resilienz für den Katastrophenfall}

\author{
Matthias Zimmer ${ }^{1,2} \cdot$ Marcel Reuter ${ }^{2,3}$ \\ ${ }^{1}$ Ketteler Krankenhaus gGmbH, Offenbach am Main, Deutschland \\ ${ }^{2}$ Abteilung 6 Bevölkerungsschutz, Berufsfeuerwehr Offenbach am Main, Offenbach am Main, Deutschland \\ ${ }^{3}$ Malteser Hilfsdienst e. V. Stadt und Kreis Offenbach, Obertshausen, Deutschland
}

\title{
Zusammenfassung
}

Hintergrund: Die Coronapandemie fordert von unserem Gesundheitssystem neue Lösungen, um in kurzer Zeit die Behandlungskapazitäten belastbar zu erweitern. Als letzte Erweiterungsstufe dient der Katastrophenschutz, dessen Durchhaltefähigkeit auch durch freiwillige Personen verbessert werden kann. Diese Spontanhelfer benötigen jedoch eine Ausbildung, um in die Hilfeleistungsstrukturen eingebunden zu werden.

Methode: Ein Expertengremium mit langjähriger Erfahrung in der Entwicklung und Durchführung von Ausbildungen entwickelte in einem stufenweisen Prozess ein Curriculum zur Ausbildung des Pandemienotfallhelfers (PNH).

Ergebnisse: Der PNH soll die vollausgebildeten Helfer während des Transports und der Versorgung in einem Behelfskrankenhaus unterstützen. Das Curriculum zur Ausbildung zum PNH umfasst 16 Unterrichtseinheiten zu je 45 min zu den Themen Einsatz, Eigenund Fremdschutz sowie unmittelbare Patientenversorgung. Im Fokus stehen praktische Fertigkeiten, für die die Teilnehmer die Durchführungsverantwortung übernehmen können.

Schlussfolgerung: Mit dem Konzept des PNH steht erstmalig eine strukturierte Vorbereitung und Eingliederung von Spontanhelfern im deutschen Katastrophenschutz zu Verfügung. Er ist kein Ersatz für vollausgebildete haupt- und ehrenamtliche Kräfte, sondern kann eine sinnvolle Unterstützung darstellen.

\section{Schlüsselwörter}

Katastrophenmedizin · Bevölkerungsschutz · Patientenversorgun · Curriculum · Spontanhelfer

Die SARS-CoV-2-Pandemie stellte das deutsche Gesundheitssystem und die öffentliche nichtpolizeiliche Gefahrenabwehr vor bislang nicht erahnte Herausforderungen. Die Limitierung der Bettenkapazität, der Mangel an geeigneter Schutzausrüstung und die Überforderung der personellen Durchhaltefähigkeit standen im Vordergrund. Ein Schlüssel zur Verbesserung der personellen Durchhaltefähigkeit könnte der Einsatz von Spontanhelfern aus der Bevölkerung sein.

Ziel dieser Arbeit ist es, das neu entwickelte Konzept des Pandemienotfallhelfers (PNH) einem breiten Fachpublikum zugänglich zu machen, um mittelfristig die Leistungs- und Durchhaltefähigkeit der organisierten nichtpolizeilichen Gefahrenabwehr durch sogenannte Spontanhelfer zu verbessern.

\section{Hintergrund}

Die teils erschreckenden Erfahrungen der weltweiten Gesundheitssysteme mit den Auswirkungen der Coronapandemie führten ab März 2020 zu der Suche nach praktikablen Lösungsansätzen, um die Resilienz der kritischen Infrastruktur "Gesundheitssystem" [11] in Deutschland punktuell zu erhöhen. Die Pandemie wurde dabei als eine ausgedehnte Schadenslage begriffen, zu deren Kontrolle Maßnahmen auch 
außerhalb der gewohnten Krankenhausstrukturen etabliert werden mussten. Dies konnte bspw. durch ein Behelfskrankenhaus für leicht erkrankte Patienten oder die Verstärkung der Transportkapazitäten erfolgen, wie es u.a. der Pandemieplan Hessen in stufenweiser Eskalation der Maßnahmen vorsah [10].

Die Strategie der nichtpolizeilichen öffentlichen Gefahrenabwehr setzte dabei auf eine Mischung aus spezialisierten haupt- und ehrenamtlichen Kräften privater oder öffentlicher Einheiten und Einrichtungen. Sie hatten nach den jeweiligen Vorschriften des Brandschutzes, des Rettungsdiensts, des Katastrophenschutzes (KatS) oder des Zivilschutzes standardisierte Ausbildungen durchlaufen. Sie verfügten über Schutzausrüstung, trainierten regelmäßig mit den zur Verfügung stehenden technischen Geräten und waren hierarchisch gegliedert. Ihnen konnte ein konkreter Einsatzwert und eine fallabhängige Toleranz gegenüber Gefahren zugesprochen werden.

Neben den haupt- und ehrenamtlichen Kräften engagierten sich in den vergangenen Jahrzehnten wiederholt nicht an Institutionen gebundene Bürger bei Katastrophen in der Bundesrepublik (Elbhochwasser 2002 23.000 Personen, Elbhochwasser 201312.000 Personen; [2]). Diese sogenannten Spontanhelfer, auch „physical volunteers" genannt [8], leisten freiwillig und ohne vorherige Bindung an die Strukturen der öffentlichen Gefahrenabwehr Unterstützung. Eine berufliche Vorbildung ist für das Engagement keine Voraussetzung, vielmehr ist das Verhalten dieser Menschen von einer hohen Motivation geprägt, aktiv zu werden [3]. Spontanhelfer können auch eine medizinische Vorbildung besitzen, wie bspw. medizinische Fachangestellte, Pflegekräfte inkl. Branchenwechsler oder aus dem aktiven Dienst ausgeschiedene Rettungsdienstmitarbeiter, Soldaten oder Katastrophenschützer. Damit kann ihr jeweiliger Einsatzwert sehr unterschiedlich sein. Unter Umständen besteht aufgrund der fehlenden medizinischen Vorbildung in der direkten Patientenversorgung kein Einsatzwert.

\section{Ausgangslage}

Die landesspezifischen Katastrophenschutzgesetze kennen die Verpflichtung der Bevölkerung zur Unterstützung. Beispielhaft sei der § 49 Abs. 1 des Hessisches Brand- und Katastrophenschutzgesetzes mit einer Hilfeleistungspflicht der Bevölkerung genannt [4]. Personen über 18 Jahre sind zu Hilfeleistungen heranzuziehen, um eine unmittelbar bevorstehende Gefahr abzuwehren. Bei der Verpflichtung sind gleichzeitig die Vorgaben des Art. 3 Abs. 3 GG (allgemeine und spezielle Gleichheitssätze) zu wahren. Die Hilfeleistungspflicht ist jedoch limitiert, wenn eine erhebliche Gefahr für den Verpflichteten droht. Aus dem juristischen Verständnis heraus schränkt also die Wahrscheinlichkeit für den Eintritt einer Schädigung des Verpflichteten dessen Einsatzmöglichkeiten ein [7]. Im Bereich des Sanitätswesens liegen neben körperlichen (Heben, Tragen etc.) und psychischen Belastungen (Leiden und Zustandsverschlechterung der Patienten, Wahrnehmung der eigenen Gesundheitsgefahr) durch die Pandemielage vor allem infektiologische Gefahren für die Helfenden vor. Im Allgemeinen sind solche Verpflichtungen bislang in der Bundesrepublik mangels verfügbarer Dokumentation nicht mit Zahlen belegbar.

Für den Einsatz von spontanen und verpflichteten Helfern bestehen rechtliche Rahmenbedingungen, die ihre Tätigkeit flankieren. So sind der Schutz der Menschenwürde (Art. 1 Abs. $1 \mathrm{GG}$ ) und das Recht auf Leben sowie körperliche Unversehrtheit (Art. 2 Abs. 2 S. 1) zu achten. Hieraus abgeleitet darf ein Helfer nicht einer erheblichen Gefahr ausgesetzt werden (siehe auch § 49 Abs. 1 HBKG).

Daher verlangt die Fürsorgepflicht des Staates gegenüber dem helfenden und dem erkrankten Staatsbürger gleichermaßen, dass die freiwilligen Helfer für ihre Aufgaben vorbereitet werden, wenn sie nicht bereits aus anderen beruflichen oder privaten Gründen über die notwendigen Kenntnisse verfügen [12]. Natürlich steigert sich auch der Einsatzwert eines Helfers immer, wenn er eine vorbereitende Schulung erhält. Doch für eine Pandemielage wie aktuell durch SARS-CoV-2 ausgelöst bestanden bislang keine Konzepte zur strukturierten Einbindung zuvor nicht organisierter Helfer.

\section{Methoden}

Ein ad hoc gebildetes Expertengremium definierte in einem mehrstufigen, zweiwöchigen Prozess die Einsatzmöglichkeiten und konsekutiv die Unterrichtsinhalte für Spontanhelfer in einem Pandemieszenario. Die Mitglieder des Expertengremiums kamen aus den Bereichen Ausbildung Erste Hilfe, Ausbildung Sanitäts- und Rettungsdienst, Einheitsführer im KatS und der Gruppe leitender Notarzt. Sie verfügten über fünf bis 20 Jahre Erfahrung in der notfallmedizinischen Ausbildung inkl. Ausgestaltung, Planung und Umsetzung von Kursinhalten für den Sanitätsdienst, den KatS und Krankenhäuser. Als Vorlagen für die Struktur und Inhalte dienten die vorhandenen Ausbildungskonzepte des KatS.

\section{Ergebnisse}

Die Führungskräfte formulierten zunächst vier Voraussetzungen für den Einsatz der Spontanhelfer in ihren Einheiten:

- Mitwirkung an der Auftragserfüllung

- Integration in bestehende Strukturen

- Minimierung der Eigengefährdung

- Minimierung der Fremdgefährdung

Dabei sollen die Spontanhelfer in den regulären taktischen Einheiten des KatS (Zug, Gruppe oder Trupp) eingegliedert werden, damit ihre Führung ohne die Schaffung neuer Führungsstrukturen auskommt. Eigene Führungsverantwortung für Spontanhelfer war nicht vorgesehen, da der personelle Engpass ausschließlich in der Patientenversorgung befürchtet wurde.

Im nächsten Schritt konnten vier Einsatzbereiche für die Spontanhelfer identifiziert werden, in denen sie die organisierten Helfer aus Haupt- und Ehrenamt unterstützen könnten: Patiententransport, Patientenversorgung, Logistik und Dokumentation.

Das Expertengremium sah eine Teilnahme aller Spontanhelfer an diesem Kurs vor, um einen einheitlichen Informationsstand zu erreichen. Zudem wurde die Festlegung getroffen, dass die medizinisch nicht vorgebildeten Spontanhelfer nur subsidiär für die organisierten und ausgebilde- 


\begin{tabular}{|l|l|}
\hline $\begin{array}{l}\text { Tab. } 1 \text { Beispiele für die Einsatzbereiche der Spontanhelfer zur Entlastung der organisierten Hel- } \\
\text { fer }\end{array}$ \\
\hline $\begin{array}{l}\text { Patienten- } \\
\text { transport }\end{array}$ & Unterstützung bei: \\
\cline { 2 - 2 } & - Innerklinischer Patiententransport von und zu Untersuchungen \\
\cline { 2 - 2 } & - Zimmerwechsel \\
\cline { 2 - 2 } & - Aufnahme oder Entlassung aus der stationären Versorgung \\
\hline \multirow{2}{*}{$\begin{array}{l}\text { Patienten- } \\
\text { versorgung }\end{array}$} & Erhebung der Vitalparameter auf Station \\
\cline { 2 - 2 } & $\begin{array}{l}\text { Springerdienst für Materialanforderungen der Pflege/Ärzte, die in einem } \\
\text { Isolierzimmer arbeiten }\end{array}$ \\
\hline \multirow{2}{*}{ Logistik } & Unterstützung der Warenwirtschaft \\
\cline { 2 - 2 } & Verteilung von Verbrauchsmaterial auf Station/Patientenzimmern \\
\cline { 2 - 2 } & Austeilen und Einsammeln der Patientenverpflegung \\
\hline Dokumentation & Patientenverwaltung \\
\cline { 2 - 2 } & Personalverwaltung \\
\hline
\end{tabular}

ten Kräfte agieren sollten (•Tab. 1). Ein vollkommen selbstständiges Arbeiten im Einsatz war nicht angedacht. Somit musste nur eine eingeschränkte Handlungskompetenz erreicht werden, differenzierte Kompetenzebenen [9] waren nicht angestrebt. Im Zentrum sollten patientennahe, manuelle und technische Fertigkeiten stehen, vergleichbar zum Nationalen Kompetenzbasierten Lernzielkatalog Medizin (NKLM; [5]). Transferleistungen wurden nicht verlangt. Sollten die Spontanhelfer über eine medizinische Vorbildung verfügen, so sollte der Freiheitsgrad inrer Tätigkeit dem Grad ihrer Vorbildung entsprechen. Die Ausbildung sollte in diesem Fall grundlegende Kenntnisse und Fähigkeiten vermitteln oder auffrischen und Unterstellungsverhältnisse eindeutig klären. Am Ende der Ausbildung sollten alle Teilnehmer die Durchführungsverantwortung für die ihnen übertragenen Aufgaben sicher übernehmen können. Am Beispiel der Blutdruckmessung nach Riva-Rocci sei dies verdeutlicht. Der Lehrgangsteilnehmer muss für die korrekte Durchführung nicht zwangsläufig wissen, dass die zu hörenden pulssynchronen Geräusche Korotkow-Geräusche heißen oder durch welche physikalischen Phänomene sie verursacht werden. Vielmehr liegt die Aufmerksamkeit auf einem fokussierten Training der manuellen Fertigkeiten.

Auf Basis der vorgenannten Bedingungen wurden die Eckpunkte zur Ausgestaltung des Unterrichts festgelegt:

- Hoher Praxisbezug der Lerninhalte

- Hintergrundwissen vermitteln, wenn es absolut notwendig für die sichere Durchführung einer Maßnahme ist
- Praktische Fähigkeiten in Kleinstgruppen (max. drei Personen) unterrichten

- Häufige Wiederholung manueller

- Beurteilung der Einsatzeignung der Spontanhelfer während der Ausbildung

Als Bewertungsgrundlage für den letzten Punkt sollen die vier von den Führungskräften formulierten Voraussetzungen dienen. Zusätzlich legte das Expertengremium fest, dass die Spontanhelfer eine primäre psychosoziale Prävention [6] erhalten müssen. Freiwillige, die während der Ausbildung seitens der Dozenten als ungeeignet identifiziert worden wären, hätten die Ausbildung nicht abschließen dürfen und wären nicht eingesetzt worden. Die Entscheidung hierüber wäre im Rahmen einer täglichen, Dienstbesprechung der Dozenten erfolgt.

Im weiteren Diskurs wurden drei universelle Themenfelder mit insgesamt zehn Unterthemen als Gerüst des Curriculums identifiziert (-Tab. 2). Das Expertengremium erstellte abschließend unter Einbeziehung aller vorgenannten Punkte einen 16 Unterrichtseinheiten à 45 min umfassenden Lehrplan mit konkreten Inhalten und Zielen (- Tab. 3). Die Ermittlung des Zeitbedarfs ergab sich aus langjährigen Erfahrungen mit der Ausbildung in Sanitätsdienst und KatS, den bekannten Zeitansätzen bestehender Ausbildungskonzepte im KatS und der gesetzten Notwendigkeit, die Spontanhelfer möglichst rasch in den realen Einsatz bringen zu können.

Der Kursleiter für das lokal geplante Konzept besaß u.a. die Qualifikation eines Ausbilders in Erste Hilfe und Multiplikators Prozeduren
Tab. 2 Universelle Themenfelder und deren Unterthemen

\begin{tabular}{|c|c|}
\hline \multirow[t]{3}{*}{ Einsatz } & Coronavirus \\
\hline & Einsatzregeln \\
\hline & Rechtskunde \\
\hline \multirow{2}{*}{$\begin{array}{l}\text { Eigen- und } \\
\text { Fremdschutz }\end{array}$} & Hygiene \\
\hline & Psychosoziale Prävention \\
\hline \multirow[t]{5}{*}{$\begin{array}{l}\text { Patienten- } \\
\text { versorgung }\end{array}$} & $\begin{array}{l}\text { Vitalwerterhebung \& -be- } \\
\text { wertung }\end{array}$ \\
\hline & Medikamente \\
\hline & Heben und Tragen \\
\hline & Nahrungsaufnahme \\
\hline & Ausscheidungen \\
\hline
\end{tabular}

der standardisierten CBRN-Grundausbildung (Bundesakademie für Bevölkerungsschutz und Zivile Verteidigung [BABZ]) sowie die ärztliche Zusatzbezeichnung Notfallmedizin. Die Unterrichtenden der Kleingruppen verfügten über die Qualifikationen Notfallsanitäter, Praxisanleiter für Notfallsanitäter oder Gruppenführer im medizinischen KatS. Die Ausbildung zum Gruppenführer beinhaltet ein Grundlagenseminar der Didaktik und die medizinische Mindestqualifikation des Rettungssanitäters.

Der Aufruf zur Suche nach Freiwilligen für die Tätigkeit als PNH wäre über die öffentlichen, lokalen Medien und die sozialen Medien in Analogie zu den angebotenen Tätigkeiten im Einkaufsdienst, Fahrdienst oder Impfzentrum erfolgt. Die interessierten Spontanhelfer hätten sich dann über eine zentrale E-Mail-Adresse bzw. Telefonnummer der ausbildenden Stelle melden können. Als Ausbildungsstätte hätte ein großer Saal einer Pfarrgemeinde gedient. Das notwendige Ausbildungsmaterial entsprach dem üblichen Inventar der basalen sanitätsdienstlichen Ausbildung inkl. des Infektionsschutzes.

Die Ausbilder des PNH sollten neben einer didaktischen Grundqualifikation über die medizinische Qualifikation Rettungssanitäter, Notfallsanitäter oder examinierte Gesundheits- und Krankenpflege verfügen und im Umgang mit Infektionsschutzkleidung gut geschult sein. Eine Grundausbildung im Bereich der standardisierten CBRN-Ausbildung des Bundes ist wünschenswert. 
Tab. 3 Curriculum des Pandemienotfallhelfers $(P N H)$

\begin{tabular}{|c|c|c|}
\hline Thema & Inhalt & $\begin{array}{l}\text { UE } \\
\text { à } 45 \mathrm{~min}\end{array}$ \\
\hline \multirow[t]{12}{*}{ Einführung } & Befähigungen und Einsatzmöglichkeiten des PNH & \multirow[t]{12}{*}{1} \\
\hline & - Wertschätzung gegenüber dem engagierten Bürger & \\
\hline & - Aufgaben mit und ohne Vorkenntnisse & \\
\hline & - Beispielhafte Einsatzmöglichkeiten & \\
\hline & Rechtskunde mit schriftlicher Belehrung über & \\
\hline & $-\S 203 \mathrm{StGB}$ & \\
\hline & $-\S 323 c$ StGB & \\
\hline & - $\S \S 53,53 a$ StPO und $\S \S 383$ ZPO & \\
\hline & Helferrechte und-pflichten & \\
\hline & $\begin{array}{l}\text { - Auszug aus dem jeweiligen Landeskatastrophenschutzgesetz } \\
\text { bzgl. des Helferrechts }\end{array}$ & \\
\hline & - Versicherung der Helfer bei Arbeits- und Wegeunfällen & \\
\hline & - Unterstellungsverhältnisse & \\
\hline \multirow[t]{9}{*}{ Coronavirus } & Biologische Eigenschaften des SARS-CoV-2 ${ }^{\mathrm{a}}$ & \multirow[t]{9}{*}{1} \\
\hline & Anerkannte Übertragungswege des SARS-CoV-2 ${ }^{\mathrm{a}}$ & \\
\hline & Die Erkrankung COVID-19a & \\
\hline & - Symptome & \\
\hline & - Mögliche Krankheitsverläufe & \\
\hline & - Komplikationen & \\
\hline & Bekämpfung ${ }^{\mathrm{a}}$ & \\
\hline & - Allgemeine Therapieoptionen & \\
\hline & - Isolationsmaßnahmen & \\
\hline \multirow[t]{18}{*}{ Hygiene } & Allgemeine Verhaltensregeln im Gesundheitsdienst & \multirow[t]{18}{*}{5} \\
\hline & - Vor, während und nach dem Dienst & \\
\hline & Hautpflege & \\
\hline & - Hautschutzplan & \\
\hline & $\begin{array}{l}\text { - Hautpflegeprodukte für die Zeiten vor, während und nach dem } \\
\text { Dienst }\end{array}$ & \\
\hline & Haut- und Oberflächendesinfektion & \\
\hline & - Hygieneplan & \\
\hline & - Produkte und Einwirkzeiten & \\
\hline & - Fallstricke & \\
\hline & - Anwendung gemäß DIN EN 1500 & \\
\hline & Persönliche Schutzausrüstung (PSA) ${ }^{\mathrm{a}}$ & \\
\hline & - Aufgabe der Ausrüstung & \\
\hline & $\begin{array}{l}\text { - Orientierende Übersicht über die Fähigkeiten der Schutzkittel, } \\
\text { Untersuchungshandschuhe und Schutzmasken }\end{array}$ & \\
\hline & - Korrektes Anlegen der PSA & \\
\hline & - Sicheres Ablegen der PSA & \\
\hline & Arbeiten im kontaminierten Bereich ${ }^{\mathrm{a}}$ & \\
\hline & - Schwarz/Weiß bzw. Rot/Gelb/Grün & \\
\hline & - Spezielle Verhaltensregeln & \\
\hline
\end{tabular}

\section{Diskussion}

Große und zeitlich lange Einsatzlagen werden seitens der Bevölkerung seit jeher mit Aufmerksamkeit begleitet. Die Bündelung der bürgerlichen Spontanbereitschaft und die Einbindung verpflichteter
Bürger können wichtige Ressourcen bei der Erfüllung des Einsatzauftrags des Katastrophenschutzes darstellen.

Mit dem Aufbau und der Nutzung eines Behelfskrankenhauses für leicht erkrankte Patienten mit SARS-CoV-2-Infektion hätte der hessische Katastrophenschutz Neu- land betreten. Obwohl in der Theorie aus den Zeiten des Kalten Krieges entsprechende Konzepte vorhanden waren, sind die personellen und materiellen Strukturen nicht mehr vorhanden. Zugleich stünden kaum oder keine examinierten Pflegekräfte oder Notfallsanitäter zur Verfügung, da sie kein Teil der Personalressourcen des medizinischen KatS sind.

Die mögliche Konzeptionierung der Ausbildung zum PNH erstreckt sich über ein großes Kontinuum beginnend bei der Auslassung einer eigenen Ausbildung bis hin zur Vollausbildung (VA) im med. KatS. Letztere würde je nach Hilfsorganisation ca. 133 Unterrichtseinheiten ohne Funk- und Kraftfahrerausbildung [13] umfassen und trotzdem kaum pflegerische Ausbildungsanteile beinhalten, die für die Versorgung stationärer Patienten notwendig wären. Auch bestünden nur basal Fähigkeiten zum Umgang mit i.v.Medikamenten und der Assistenz anderer Maßnahmen. Die alleinigen Kräfte des KatS sind nur bedingt für den Betrieb eines Behelfskrankenhauses geeignet. Geeigneter wäre die Ausbildung zur Schwesternhelferin/zum Pflegediensthelfer, die eine Basisqualifikation für die ambulante und stationäre Pflege darstellt und ihren Ursprung im Zivilschutz des Kalten Krieges hatte. Mit 120 UE plus $90 \mathrm{~h}$ Praktikum ist dieses Ausbildungsformat nicht geeignet, Spontanhelfer in ihrem Engagement zu kanalisieren. Da der PNH auch auf Spontanhelfer mit medizinischer Vorbildung abzielt, muss ein Kompromiss zwischen den unterschiedlichen Extremen gefunden werden. Denn orientiert man sich an den medial begleiteten Spontanhelfern der deutschen Hochwasserlagen 2021, dann ist jeweils gut erkennbar, dass Spontanhelfer rasch in einen realen Einsatz gehen und die Wirksamkeit ihrer Tatkraft bei den Hilfsbedürftigen erleben möchten. Länger dauernde Lehrgänge stehen dem entgegen und zudem ist die Dauer des Engagements meist durch die eigene Berufstätigkeit oder ggf. noch laufende Ausbildung limitiert.

Die Arbeit im KatS erfolgt üblicherweise in der Auftragstaktik. Zum Agieren in dieser Befehlsform benötigt der PNH eine Orientierung über das Einsatzgeschehen (Lageeinweisung). Dies wird im Curriculum durch Informationen über das Coronavi- 


\begin{tabular}{|c|c|c|}
\hline Thema & Inhalt & $\begin{array}{l}\text { UE } \\
\text { à } 45 \mathrm{~min}\end{array}$ \\
\hline \multirow{16}{*}{$\begin{array}{l}\text { Vitalwert- } \\
\text { erhebung }\end{array}$} & Bewusstsein & \multirow[t]{16}{*}{3} \\
\hline & - Wach, schläfrig \& ansprechbar, schläfrig \& weckbar, bewusstlos & \\
\hline & Atmung & \\
\hline & - Normale Atmung kennen & \\
\hline & - Frequenz auszählen & \\
\hline & Puls & \\
\hline & - Tasten am Handgelenk & \\
\hline & - Rekapillarisierungszeit & \\
\hline & Blutdruck & \\
\hline & $\begin{array}{l}\text { - Messung mit einer manuellen Oberarmmanschette und Stetho- } \\
\text { skop }\end{array}$ & \\
\hline & - Oszillometrische Messung am Oberarm & \\
\hline & Temperatur & \\
\hline & - Tympanale Messung & \\
\hline & Transkutane Sauerstoffsättigung & \\
\hline & - Anwendung des Messgeräts & \\
\hline & $\begin{array}{l}\text { Praxisübungen unter Einhaltung der Hygienevorschriften, bei } \\
\text { Verfügbarkeit auch mit Simulationsphantomen }\end{array}$ & \\
\hline \multirow[t]{17}{*}{$\begin{array}{l}\text { Kritische } \\
\text { Vitalwerte }\end{array}$} & $\begin{array}{l}\text { Grenzwerte, bei denen sofort ein medizinischer Vorgesetzter infor- } \\
\text { miert werden muss: }\end{array}$ & \multirow[t]{17}{*}{0,5} \\
\hline & Bewusstsein & \\
\hline & - Nicht wach & \\
\hline & Atmung & \\
\hline & - Atemfrequenz unter $10 / \mathrm{min}$ und über $21 / \mathrm{min}$ & \\
\hline & - Nicht normale Atmung & \\
\hline & Puls & \\
\hline & - Unter $50 / \mathrm{min}$ und über $100 / \mathrm{min}$ & \\
\hline & - Unregelmäßiger Puls & \\
\hline & Blutdruck & \\
\hline & - Systolisch unter $100 \mathrm{~mm} \mathrm{Hg}$ und über $150 \mathrm{~mm} \mathrm{Hg}$ & \\
\hline & - Diastolisch unter $50 \mathrm{~mm} \mathrm{Hg}$ und über $100 \mathrm{~mm} \mathrm{Hg}$ & \\
\hline & Temperatur & \\
\hline & - Unter $36,0^{\circ} \mathrm{C}$ und über $38,3^{\circ} \mathrm{C}$ & \\
\hline & Transkutane Sauerstoffsättigung & \\
\hline & - Unter $90 \%$ & \\
\hline & $\begin{array}{l}\text { Generell empfehlenswert sind einlaminierte, abwischbare Karten } \\
\text { mit den Grenzwerten für jeden Helfer }\end{array}$ & \\
\hline \multirow[t]{4}{*}{ Medikamente } & Umgang mit Infusionen & \multirow[t]{4}{*}{1} \\
\hline & - Vorbereiten & \\
\hline & $\begin{array}{l}\text { - Anschließen an und Entfernen von einem peripheren Venenver- } \\
\text { weilzugang }\end{array}$ & \\
\hline & - Praxisübungen an i.v.-Zugangs-Dummies & \\
\hline \multirow{4}{*}{$\begin{array}{l}\text { Heben und } \\
\text { Tragen }\end{array}$} & Anwendung einer & \multirow[t]{4}{*}{2} \\
\hline & - Fahrtrage, Feldkrankentrage, eines Rollstuhls & \\
\hline & Hilfe beim Umlagern & \\
\hline & Unterstützen beim Gehen & \\
\hline
\end{tabular}

rus, die Einsatzregeln und rechtskundliche Aspekte abgebildet. Die Inhalte der Rechtskunde sind im Vergleich zu einer VA (10 UE) stark auf den konkreten Einsatz fokussiert. So fehlen bspw. Belehrungen über SGB, StVO oder MPG, weil die PNH hiermit keine Berührungspunkte haben sollten. Für die Informationen (eine UE) über das Coronavirus besteht vergleichsweise viel Zeit, wenn man vergleicht, dass ein einfacher Sanitäter sonst in gleicher Zeit vier neurologische Krankheitsbilder inkl. Anatomie des ZNS vorgestellt bekommt.

Die Unterweisung in die Hygiene und Gefahrstoffe (5UE) gehört zu den Grundpflichten einer Führungskraft im KatS, vgl. DGUV Vorschrift 1. In der VA werden gemeinsam mit anderen Themen hierauf 2 UE verwendet. Die Schulung auf die Verwendung der PSA findet bei der VA in eigenem Lehrgang (16UE) statt, der eine sehr hohe Detailtiefe besitzt, einen großen Anteil an Gefahrstofflehre/Einsatztaktik umfasst und nicht von jedem Sanitäter im KatS absolviert wird. Durch eine Konzentration der Inhalte ist eine Kürzung der Zeiten und der Inhalt auf den Schutz vor SARS-CoV-2 sowie die Einhaltung der allgemeinen $\mathrm{Hy}$ giene vertretbar.

Die Lerneinheiten Vitalwerte und kritische Vitalwerte $(3,5 \mathrm{UE})$ werden in der VA nicht derart prägnant präsentiert wie beim $\mathrm{PNH}$. Sie ergeben sich in der VA über die Zeit aus Anatomie und Krankheitslehre (16UE). Das Curriculum des PNH bietet im Vergleich hierzu einen einfachen und direkten Zugang zu diesem Wissen. Gerade für das Erlernen der Parametererfassung wird mehr Zeit eingeräumt als bei der VA.

Der Umgang mit Infusionen (eine UE) bewegt sich im ähnlichen Rahmen wie bei einer VA (2UE), wobei auf andere Applikationsformen verzichtet wurde, die nicht im Aufgabenbereich des PNH stehen. Dies ermöglicht eine intensivere Schulung des PNH. Der PNH ohne med. Vorbildung soll selbst keine Injektionen vornehmen, da hier eine große Gefahr für die Patientensicherheit gesehen wird. Aber weil Patienten mit Infusion zum Stationsalltag gehören, muss jeder PNH mit Infusionen umgehen können.

Für Heben und Tragen wurden $2 \mathrm{UE}$ vorgesehen, während es in der VA 6UE sind. Übungen wie Gehen mit dem Rollator, Assistenz beim Treppensteigen, Um- 


\begin{tabular}{|c|c|c|}
\hline Thema & Inhalt & $\begin{array}{l}\text { UE } \\
\text { à } 45 \mathrm{~min}\end{array}$ \\
\hline \multirow{10}{*}{$\begin{array}{l}\text { Patienten- } \\
\text { versorgung }\end{array}$} & Grundsätze der Kommunikation & \multirow{10}{*}{1,5} \\
\hline & - Kontaktaufnahme mit Hilfsbedürftigen & \\
\hline & - Verhaltensregeln im Umgang mit Hilfsbedürftigen & \\
\hline & Unterstützung beim Essen und Trinken & \\
\hline & - Grundregeln der Hygiene & \\
\hline & - Verständnis für Probleme der Nahrungsaufnahme & \\
\hline & - Praxisübungen & \\
\hline & Unterstützung bei der Notdurft & \\
\hline & - Herstellen einer Intimität & \\
\hline & - Hygienischer Umgang mit Fäkalien & \\
\hline \multirow[t]{6}{*}{ Abschluss } & Primär psychologische Prävention & \multirow[t]{6}{*}{1} \\
\hline & - Ursachen von Stress & \\
\hline & - Stresskurve: Stressreaktion, Resistenzphase, Erschöpfung & \\
\hline & - Achtsamkeit für andere und sich selbst & \\
\hline & $\begin{array}{l}\text { - Möglichkeiten und Ansprechpartner der psychosozialen Einsatz- } \\
\text { betreuung }\end{array}$ & \\
\hline & Verabschiedung & \\
\hline
\end{tabular}

gang mit dem Tragetuch oder -stuhl sind entfallen. Es können Grundfertigkeiten erworben werden, mit denen ein regulärer Helfer im KatS unterstützt werden kann. Eine längere Ausbildung wäre gerade für PNH ohne Vorbildung grundsätzlich wünschenswert, steht jedoch unter dem Zwiespalt, einen weiteren Ausbildungstag zu generieren.

Die pflegerische Patientenversorgung wurde mit 1,5 UE angesetzt. Im Gegensatz zur VA (2UE) werden bspw. keine Lagerungsmaßnahmen oder der Umgang mit einem Tracheostoma erörtert. Im Zentrum steht ein respektvoller, wertschätzender Umgang mit den Patienten, die Vermeidung der Nahrungsaspiration und der Umgang mit Exkrementen; nicht aber die eigentliche pflegerische Versorgung. Der PNH ohne med. Vorbildung soll auch hier eine Assistenz und kein Ersatz für besser qualifiziertes Personal sein.

Die primär psychologische Prävention erfolgt analog zur VA und soll die Langzeitfolgen psychisch belastender Situationen verhindern bzw. mildern. Die Dauer ist kürzer als beim Einsatzersthelfer $A$ der Bundeswehr [14] oder im Zivilschutz (2 UE; [15]). Es besteht jedoch keine Evidenz über die Dauer oder Gestaltung einer bestmöglichen primär psychologischen Prävention.
Angesichts der umrissenen Aufgaben des PNH erscheinen die angesetzten Zeiten und Inhalte vertretbar, auch wenn hierfür Abstriche beim späteren Einsatzwert in Kauf genommen werden müssen. Die Teilnahme an einem Erste-Hilfe-Kurs (9UE) mag sinnvoll erscheinen, doch viele Inhalte zielen deutlicher auf eine traumatologische Notfallversorgung ab, sodass nur ausgewählte Themen für den $\mathrm{PNH}$ relevant wären (bspw. Reanimation und Bewusstlosigkeit). Bei der Beurteilung des vorliegenden Konzepts des PNH sollte zudem bedacht werden, dass meist keine wissenschaftliche Evidenz für die Zusammensetzung der VA besteht und der Betrieb eines Behelfskrankenhauses nicht das Ziel einer VA ist.

\section{Limitierung}

Die Erstellung des Curriculums war mit zwei Wochen Dauer zeitkritisch. Komplexe Methoden wie das Delphi-Verfahren wurden daher verworfen, die unter Umständen bessere Ergebnisse ermöglicht hätten. Die Kürze des Lehrgangs ist den beschränkten Aufgaben und der Intention einer schnellen Einsetzbarkeit der Kräfte geschuldet. Es ist grundsätzlich zu überlegen, ob es ein unterschiedliches Curriculum für Spontanhelfer mit und ohne medi- zinische Vorbildung geben sollte, um den Bedürfnissen der Helfer gerechter zu werden. Auch die Verlängerung um mindestens einen weiteren halben Tag könnte zur Vertiefung ausgewählter Themen dienen, bspw. Heben und Tragen. Zugleich muss aber auch von einer eingeschränkten Ausbildungskapazität ausgegangen werden. Denn wer ausbildet, der fehlt gleichzeitig im Einsatz und die Personalressourcen sind teils sehr eng.

Zur vollständigen Erlangung der Einsatzfähigkeit der Spontanhelfer gehört die Ausstattung mit einer geeigneten Einsatzbekleidung. Hierbei können bspw. lokale Lösungen über Wäschepools der Krankenhäuser, der Rettungsdienste oder des KatS wegweisend sein.

Der Einsatzzeitraum eines PNH ist nur schwer abzuschätzen. Das vorstellbare Kontinuum reicht von einem täglichen Engagement von zwei Wochen bis zu einem längeren Einsatzzeitraum alle paar Tage. Bedingt durch das freiwillige, ehrenamtliche Engagement, das höchstwahrscheinlich durch hauptberufliche Verpflichtungen limitiert wäre, und die stattgehabte wellenförmige Belastung des Gesundheitssystems wäre der Einsatz eines einzelnen PNH dauerhaft diskontinuierlich gewesen. Ob überhaupt derselbe als PNH qualifizierte Spontanhelfer auch während einer zweiten oder dritten schweren Pandemiewelle zur Verfügung stehen würde, ist nicht zu beantworten. Im Fall eines Zweiteinsatzes im Zuge einer weiteren Pandemiewelle erschienen bedarfsgerechte Auffrischungsschulungen angebracht.

Die konkrete Abschätzung der psychischen Belastungen für den einzelnen Spontanhelfer ist ex ante nicht abzuschätzen. Die Fürsorgepflicht eines jeden Vorgesetzten im KatS gebietet, bei Erkennen einer mangelhaften Resilienz während der Ausbildung oder im Verlauf des Einsatzes entsprechend einzuschreiten. Hierbei unterscheidet sich der Spontanhelfer nicht von vielen ehrenamtlichen Helfern im KatS, die in der überwiegenden Mehrheit noch nie schwerer Erkrankte/Verletzte versorgt haben.

Zum heutigen Zeitpunkt sind die Effektivität, die Dienstzufriedenheit und somit auch die Durchhaltefähigkeit eines PNH ungewiss. Auch kann aktuell nicht 
abgeschätzt werden, welches zwischenmenschliche Verhältnis sich zwischen den spontanen und organisierten Helfern entwickeln wird. Gleichzeitig bietet das Projekt des PNH eine moderne Antwort auf die Hilfsbereitschaft einer Bevölkerung, die sich augenscheinlich eher kurzfristig entscheidet und sich nicht unbedingt langfristig an starre Strukturen (Vereine, Institutionen) binden möchte [1]. Grundsätzlich ist es denkbar, für andere Szenarien (Bergrutsch, Hochwasser, Sturmfluten etc.) des Bevölkerungsschutzes das vorliegende Konzept modular zu adaptieren. Die Notwendigkeit, die Ausrichtung und der Umfang solcher Adaptionen obliegen den Beurteilungen der jeweiligen Fachdienste der Gefahrenabwehr. Das Konzept des Pandemienotfallhelfers hat seine Nagelprobe noch vor sich.

\section{Fazit für die Praxis}

- Die Resilienz des Gesundheitssystems und des Katastrophenschutzes könnte durch kanalisierte Spontanhelfer erhöht werden, welche die haupt- und ehrenamtlichen Einsatzkräfte in konkreten Punkten unterstützen.

- Die Spontanhelfer bedürfen einer einheitlichen Kurzausbildung, um sie sicher und rasch in das Gefüge der Gefahrenabwehr zu integrieren. Dabei unterliegt die Ausbildung einer stringenten OutcomeOrientierung.

- Wie auch für alle anderen Einsatzkräfte sollte eine primäre psychosoziale Prävention vor dem eigentlichen Einsatz erfolgen.

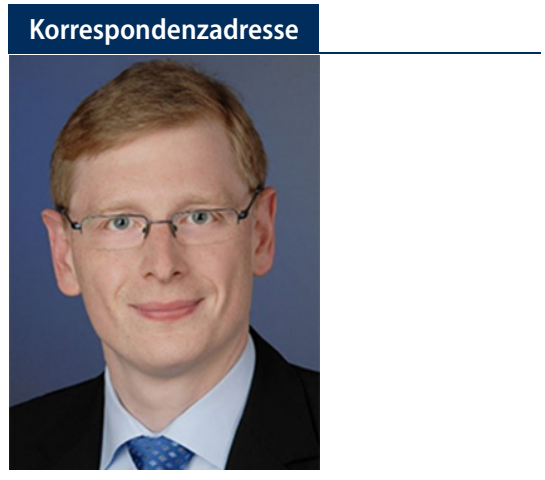

Dr. med. Matthias Zimmer

Ketteler Krankenhaus gGmbH

Lichtenplattenweg 85, 63071 Offenbach am

Main, Deutschland

zimmer.m@ketteler-krankenhaus.de

\section{Pandemic relief volunteers-increasing resilience for disasters}

Background: The corona pandemic demands new solutions from our health care system in order to expand treatment capacities in a resilient manner within a short period of time. The last stage of expansion is disaster protection, the resilience of which can also be improved by volunteers. However, these spontaneous volunteers require training in order to be integrated into the disaster relief structures.

Methods: In a step-by-step process, an ad hoc expert panel developed a curriculum for pandemic relief volunteer (PRV) training.

Results: The goal of PRV is to assist fully trained responders during transport and care in a makeshift hospital. The curriculum for training as a PRV comprises 16 instructional units of $45 \mathrm{~min}$ each on the topics of deployment, self-protection, protection of others, and direct patient care. The focus is on practical skills for which the participants can take responsibility for execution.

Conclusion: The concept of the PRV is the first structured training and integration of spontaneous responders in German civil protection. It is not a substitute for fully trained full-time and voluntary staff, but can provide useful support.

\section{Keywords}

Disaster medicine - Civil protections · Patient care · Curriculum · Spontaneous voluntee

\section{Einhaltung ethischer Richtlinien}

Interessenkonflikt. M. Zimmer und M. Reuter geben an, dass kein Interessenkonflikt besteht.

Für diesen Beitrag wurden von den Autoren keine Studien an Menschen oder Tieren durchgeführt. Für die aufgeführten Studien gelten die jeweils dort angegebenen ethischen Richtlinien.

\section{Literatur}

1. Bücherl G (2014) Wir packen an! Team Bayern organisiert Laienhelfer im Katastrophenfall. Bevölkerungsschutz 2:38

2. Erkens H (2016) Rechtliche Koordinaten für den Einsatz von Spontanhelfern. Bevölkerungsschutz 2:28-32

3. Fathi R (2016) Die Rolle von ungebundenen Helferlnnen bei der Bewältigung von Schadensereignissen. Teil 3: Handlungs- und Umsetzungs empfehlungen für den Einsatz ungebundener Helferlnnen. Deutsches Rotes Kreuze. V., Berlin

4. Hessisches Gesetz über den Brandschutz, die Allgemeine Hilfe und den Katastrophenschutz (Hessisches Brand-und Katastrophenschutzgesetz - HBKG) in der Fassung der Bekanntmachung vom 14. Januar 2014 (GVBI. S. 26), zuletzt geändert durch Gesetz vom 23. August 2018 (GVBI. S. 374)

5. MFT Medizinischer Fakultätentag der BundesrepublikDeutschland e. V. (2015) Klinisch-praktische Fertigkeiten. In: Nationaler Kompetenzbasierter Lernzielkatalog Medizin. MFT Medizinischer Fakultätentag der Bundesrepublik Deutschland e. V., Berlin, S148-158

6. Krüsmann M, Karl R, Schmelzer M, Butollo W (2006) Primäre und Sekundäre Prävention im Einsatzwesen Zusammenfassung der Ergebnisse - Darstellung einer Gesamtkonzeption. LudwigMaximilians-Universität, München

7. Paragraf 34 Rn. 58 (2016) In: Joecks W, Miebach K (Hrsg) Münchener Kommentar zum Strafgesetz- buch: StGB, Band 1: §§ 1-37. Verlag C. H. Beck, München

8. Sackmann S, Lindner S, Gerstmann S, Betke H (2018) Einbindung ungebundener Helfer in die Bewältigung von Schadensereignissen. In: Reuter C (Hrsg) Sicherheitskritische Mensch-ComputerInteraktion. Springer Vieweg, Wiesbaden

9. Schmidt HG, Moust JHC (2000) Towards a taxonomy of problems used in problem-based learning curricula. JExcell Coll Teach 11(2):57-72

10. Sonderschutzplan 4 im Aufgabenbereich 5 Sanitätswesen: Pandemieplan des Landes Hessen, Stand Februar 2007, S21-30

11. Voßschmidt S, Karsten A (2019) Grundlagen und Begriffe. In: Voßschmidt S, Karsten A (Hrsg) Resilienz und Kritische Infrastrukturen. Kohlhammer, Stuttgart, S30-38

12. Walus A (2013) Dienst- und Hilfeleistungsverpflichtungen zur Sicherstellung des Bevölkerungsschutzes. https://opinioiuris.de/aufsatz/3049\# Reichweite_einer_Eigengefaehrdungspflicht. Zugegriffen:6. Apr. 2020

13. Malteser Hilfsdienst e. V. (2016) Ausbildungsvorschrift 10. Malteser Hilfsdienste. V., Köln

14. Sanitätsamt der Bundeswehr (2010) Ausbildungsweisung Nr. 804 915, Einsatzersthelfer A. Sanitätsamt der Bundeswehr, München

15. Ergänzende zivilschutzbezogene Ausbildung der Einheiten und Einrichtungen des Katastrophenschutzes nach Landesrecht gem. §11 Abs. 1 i. V.m. $\S 13$ Abs. 4 des Gesetzes über den Zivilschutz und die Katastrophenhilfe des Bundes. Bundesamt für Bevölkerungsschutz und Katastrophenhilfe. 2018 Bonn 
Hier steht eine Anzeige.

黑 Springer 